\title{
Functional adaptation of tendon and skeletal muscle to resistance training in three patients with genetically verified classic Ehlers Danlos Syndrome
}

\author{
Mathias Bech Møller \\ Michael Kjær \\ René Brüggebusch Svensson \\ Jesper Lovind Andersen \\ Stig Peter Magnusson \\ Rie Harboe Nielsen
}

Institute of Sports Medicine Copenhagen, Department of Orthopedic Surgery M, Bispebjerg Hospital and Centre for Healthy Aging, Faculty of Health and Medical Sciences, University of Copenhagen, Denmark

Corresponding author:

Mathias Bech Møller

Institute of Sports Medicine Copenhagen, Department of Orthopedic Surgery M

Bispebjerg Hospital and Centre for Healthy Aging, Faculty of Health and Medical Sciences,

University of Copenhagen

Bispebjerg Bakke 23

2400 Copenhagen NV, Denmark

E-mail: mathiasbechmoeller@gmail.com

\section{Summary}

Background: tendon and skeletal muscle function adapts to physical training of resistive nature, but it is unknown to what extent persons with genetically altered connective tissue - who have a higher than normal tendon extensibility - will obtain any effect upon their tendon and muscle when undergoing muscle strength training. We investigated patients with classical Ehlers Danlos Syndrome (EDS) (collagen type $V$ defect) who display articular hypermobility, skin extensibility and tissue fragility.

Methods: subjects underwent strength training 3 times a week for 4 months and were tested before and after intervention in regards to muscle strength, tendon mechanical properties, and muscle function.

Results: three subjects completed the scheduled 48 sessions and had no major adverse events. Mean isometric leg extension force and leg extensor power both increased by 8 and $11 \%$ respectively (358 to $397 \mathrm{~N}$, and 117 to $123 \mathrm{~W}$ ). The tendon stiffness was tested and an average increase in response to physical training, from 1795 to $2519 \mathrm{~N} / \mathrm{mm}$ was found. On average, the training loads both in upper and lower body exercises increased by around $30 \%$ over the training period. When testing balance, the average sway-area of the participants decreased by $26 \%(0.144$ to 0.108 $\mathrm{m}^{2}$ ). On the subscale of CIS20 the participants lowered their average subjective fatigue score from 33 to 25.

Conclusion: in this small pilot study, heavy resistance training was both feasible and effective in classic Ehlers Danlos patients, and the results indicated that both tendon and skeletal muscle properties can be improved also in this patient group when they are subjected to resistance training.

KEY WORDS: CIS20, patellar tendon, resistance training, sway.

\section{Introduction}

In healthy individuals, heavy resistance training can improve both skeletal muscle mass and function ${ }^{1}$ as well as increase the stiffness of tendon structures ${ }^{2}$. Conversely, immobilization or inactivity is associated with a marked decline in skeletal muscle mass, muscle strength and tendon stiffness in both young and elderly individuals ${ }^{3-5}$.

Ehlers Danlos Syndrome (EDS) is a heterogeneous group of heritable connective tissue disorders characterized by articular hypermobility, skin extensibility and tissue fragility ${ }^{6}$. In the classical type of EDS that comprises $50-90 \%$ of all cases $^{7,8}$, a genetic defect in collagen type $\mathrm{V}$ is the most common cause ${ }^{9}$. Recently it was found that patellar tendon stiffness was significantly lower in classic EDS patients when compared to a healthy control group ${ }^{10}$. Over extensibility of the connective tissue and the accompanying joint hypermobility can lead to dislocation and habitual subluxation of the joints and also cause joint instability ${ }^{11,12}$. Joint instability can in turn lead to severe pain, which subsequently is associated with functional impairment $^{13}$. In a cross sectional study $76 \%$ of classical EDS patients reported having pain on the McGill Pain Questionnaire. Further, Voermans et al., 2011 showed that there is a positive relationship between fatigue severity and muscle weakness in EDS ${ }^{14}$. We were therefore - not only from a basic physiological point of view but also from a clinical perspective - in- 
terested in investigating whether strength training would positively influence the musculo-tendinous properties in patients with EDS. We investigated this by subjecting a group of classic EDS patients to heavy resistance training and determined muscle and tendon characteristics before and after a 4 months training period. The hypothesis was that if the patients could withstand the training it would positively influence muscle strength and elevate tendon stiffness.

\section{Methods}

\section{Subjects}

At total of 9 subjects were initially contacted and gave informed consent to participate in the study, which was approved by the Ethics Committee of Capital Region of Denmark (H-1-2013-044). The characteristics of all the initial participants are shown in Table 1. The inclusion criteria were diagnosis with the classic type of EDS (cEDS), hypermobility type of EDS (htEDS), or Benign Joint Hypermobility Syndrome (BJHS) by their own physician or specialist and patients had to be at least 18 years of age. The exclusion criteria were severe knee problems or diagnosis with vascular type of EDS. Participants were contacted and interviewed at the facility. Four subjects were diagnosed with cEDS, two with htEDS and three with BJHS. Three subjects dropped out before the beginning of the training sessions. Additionally three participants dropped out after 1-2 low-load training sessions because they found the project and transportation too time demanding, and not due to any side-effects during or as a result of the training. Consequently, 3 participants completed all required training sessions. The 3 remaining participants all had genetically verified cEDS.

The participants were subjected to a test battery with tests grouped into three test days. The participants completed a total of 48 training sessions, and were tested by the same procedure as before the intervention.

\section{Training protocol}

The training carried out by the participants was progressive resistance training 3 days a week on Mondays, Wednesdays, and Fridays ensuring 48 hours of restitution between sessions ${ }^{15}$.

The training protocol consisted of two parts. The first part was targeting the lower body and the second targeting the upper body. The two parts were carried out in continuation with the lower body part always being the first. The specific exercises were: leg extension, leg curl, leg press, resisted calf raises, chest press, seated rows, abdominal crunch and lower back extension. Before each session, the participants started out with 5 minutes warm-up on a stationary bike. The intensity was self-chosen and without progression. The upper body protocol always consisted of 4 exercises, 3 sets per exercise and aimed at a mean of 10 repetitions per set. The weights were adjusted in the same way in the lower and upper body protocol. The weight was adjusted when the participants were able to complete two or more extra repetitions per set on average. In the first training session the 10 Repetition Maximum (RM) for the calf raises and upper body exercises were estimated. This was only done to estimate the load at which the participants were able to perform the exercises properly. Before beginning the training the participants had their strength tested in the three main lower body exercises. A 5 RM test was carried out and used to predict the $10 \mathrm{RM}$ training load. First the $1 \mathrm{RM}$ was estimated from the $5 \mathrm{RM}$ test and subsequently the $10 \mathrm{RM}$ was estimated from the $1 \mathrm{RM}$. This was done in order to use the Brzycki formula ${ }^{16}$. A familiarization period was introduced in the first 12 training sessions. This period introduced the participants to the strength training ensuring cor-

Table 1. Participant characteristics.

\begin{tabular}{lllllll}
\hline & Sex & Age & Diagnosis & Drop out & Sessions & Reason \\
\hline Participant 1 & F & 42 & cEDS & - & 48 & - \\
\hline Participant 2 & F & 64 & cEDS & - & 48 & - \\
\hline Participant 3 & M & 28 & cEDS & - & 48 & - \\
\hline Participant 4 & M & 38 & BJHS & x & 0 & Did not have the time \\
\hline Participant 5 & F & 43 & cEDS & x & 0 & Too much transport \\
\hline Participant 6 & F & 48 & BJHS & x & 2 & Transportation \\
\hline Participant 7 & F & 32 & BJHS & x & 1 & Did not have the time \\
\hline Participant 8 & F & 55 & htEDS & x & 0 & Knee surgery* \\
\hline Participant 9 & M & 22 & htEDS & x & 1 & Did not have the time \\
\hline
\end{tabular}

$\mathrm{x}$ indicates dropout. Sessions is the number of training sessions completed before dropping out.

* Participant 8 accepted a pre-examination for knee-surgery shortly after inclusion. cEDS, classic Ehlers Danlos Syndrome; BJHS, Benign Joint Hypermobility Syndrome; htEDS, hypermobility type Ehlers Danlos Syndrome. 
Functional adaptation of tendon and skeletal muscle to resistance training in three patients with genetically verified classic Ehlers Danlos Syndrome

Table 2. Targeted progression in the lower body protocol.

\begin{tabular}{llllllll}
\hline Session & $1-12$ & $13-16$ & $17-24$ & $25-32$ & $33-39$ & $40-45$ & $46-48$ \\
\hline Nr. of sets & 3 & 3 & 4 & 4 & 5 & 5 & $3^{*}$ \\
\hline Repetitions & $10,10,10$ & $10,9,8$ & $10,9,8,7$ & $9,8,7,6$ & $10,9,8,7,6$ & $9,8,7,6,5$ & $6,6,6$
\end{tabular}

* In the last sessions a tapering period were carried out with the same weight but lower number of sets and reps. The tapering period in the last 3 sessions were introduced to allow for the anabolic response.

rect technique before advancing to heavier loads, thereby diminishing the risk for harmful events and side effects associated with training. In the familiarization period the participants carried out 3 sets of 10 repetitions. They began at $50 \%$ of $10 \mathrm{RM}$ in the first week, $70 \%$ in the second week, $90 \%$ in the third and in the fourth week they reached $100 \%$ of $10 \mathrm{RM}$. The participants carried out a total of 48 sessions following the progression model in Table 2. If the participants skipped 1 session, 1 was added at the end, so all participants completed the same number of sessions. The weights were continuously adjusted to the increased capabilities. If the participants reported pain while performing any of the exercises, the load was reduced and gradually increased to the intended level.

\section{Tendon mechanical properties}

The mechanical properties of the patellar tendon were tested by having the subjects do a ramped isometric contraction for 10 seconds. A strain gauge measured the developing force while an ultrasound head, aimed at the patellar tendon, simultaneously measured the elongation of the tendon. The ultrasound video and the force measures from the strain gauge were collected synchronously on a computer. From these data a force-elongation curve was constructed to estimate the stiffness of the tendon. The pre- and post-test curves were cut at the highest common force value to ensure that strain and stiffness were compared in the same part of the curve. The protocol for the measurement of tendon mechanical properties, including the reliability, has been reported previously2, 17, 18 .

\section{Isometric muscle strength}

Isometric muscle strength was measured in the muscle function evaluation chair (Metitur, Helsinki Finland $)^{19,20}$. This chair is adjustable with a fixed dynamometer that can be attached to the ankle of the participant and measure maximal isometric force production. Patients were seated upright in the chair with the hip flexed approximately at $90^{\circ}$. Seat back and leg position was adjustable in order to evaluate muscle function in the most comfortable position. The knee of the tested limb was fixed at $60^{\circ}$ flexion, which is the angle of maximal isometric quadriceps force production ${ }^{21}$. The force production was measured with a strain gauge (linear range, 0-1961 N; sensitivity, $5.1 \mathrm{mV} / \mathrm{N}$; Good Strength, Metitur, Jyvaskyla, Finland) attached to the ankle with firm Velcro straps 2 finger breadths above the top of calcaneus. All adjustable positions were noted and replicated in the post-intervention test. The participant was fixed with a belt around the pelvis and over the thigh of the tested leg. To get the feeling of the isometric contraction participants were allowed to make a couple of test contractions.

On a countdown from 5, participants were instructed to contract as fast and forcefully as possible and hold the contraction for 5 seconds. The participant carried out at least 3 attempts and more if they improved. Between attempts the participant rested for one minute. Before each attempt a gravity correction was carried out. The highest force production was used for analysis.

\section{Leg extension power}

Maximal leg extension power was measured using a Nottingham Power Rig (University of Nottingham Medical School, Model NG7 2UH, Nottingham, UK). For extensive details on method, feasibility, reliability and validity ${ }^{22}$. In short, Participants were seated in a chair build like a recumbent exercise bike. At the start, the foot of the test leg was resting on a pedal at approximately $90^{\circ}$ knee flexion and the rig was adjusted to ensure a knee angle of $15^{\circ}$ at the final position of maximal extension. The participants were instructed to push as fast and forcefully on the pedal as possible. The participants carried out at least 3 attempts and more if they improved. The participants rested one minute between each attempt. The attempt resulting in the highest power was used for analysis.

\section{Dynamic training strength}

Dynamic training load was estimated with a 5 RM test. This was done by starting out with a heavier weight then what the participants could possibly lift. Then the weight was reduced until the participants were able to carry out 5 approved repetitions. The Participants were allowed 2 minutes break between each attempt. The highest weight lifted 5 times was registered. 


\section{Functional strength}

Functional strength was estimated in a 30 second chair stand test ${ }^{23}, 24$. The participants were asked to start in a sitting position with arms folded across the chest. They were allowed a few trial-stands to adjust position of the feet to the optimal place. Participants were asked to perform as many chair stands in 30 seconds as possible. The number of finished chair stands was registered.

\section{Balance}

Postural sway was measured on a force platform (AMTI R6-1000, Watertown, MA, USA). Participant 1 and 3 completed the test on one leg and participant 2 completed the test on two legs, due to inability to keep balance on one. The participants were instructed to stand as still as possible for 30 seconds while looking at a fixed target 2.5 meters away. The test on one leg was carried out with the big toe in proximity to, but not resting on the medial malleolus. The test on two legs was carried out with the feet close together. The force platform and the appurtenant acquisition software (Matlab, Mathworks) recorded the change in center of pressure (CoP) and thereby calculated a sway area. Three consecutive trials of 10 seconds were carried out and the added data from these 3 were used as results.

\section{Fatigue}

Participants filled out the 20 item Checklist of Individual Strength (CIS20) fatigue questionnaire ${ }^{25}$. It consists of four dimensions; subjective experience of fatigue, reduction in motivation, reduction in activity and reduction in concentration. The participants filled out the questionnaire before the training and one week after they finished all training sessions and strength tests. In this way the authors hoped to detect differences in fatigue before and after the study. The total fatigue score and the score on the subscale of subjective experience of fatigue was used for further analysis.

\section{Body composition}

Before and after the intervention body composition was measured with dual-energy $\mathrm{X}$-ray absorptiometry (DEXA) scanning (Lunar DPX-IQ software version 4.6 c; Lunar, Madison, WI). The subjects were placed in the supine position, and a full body scan was performed using the medium scan mode. Because only full body measures were used, no extended research analysis was carried out. The DEXA scanner was calibrated in the morning before each participant were scanned using the recommended daily calibration procedure (Lunar "Quality Assurance").

\section{Results}

Three participants completed the training, and of the scheduled sessions they cancelled 4 out of 52,7 out of 55 , and 22 out of 70 respectively. Every missed session was added to the program ensuring that all participants completed a total of 48 session each.

\section{Biomechanical properties}

The knee extension moment was increased from 83.7 $\mathrm{N}(96.2,32.7$ and 122$)$ before the intervention to 104.2 N (104.2, 41.4 and 167) after the intervention. The maximum deformation measured at the highest common force (for the individual) decreased from $2.25 \mathrm{~mm}(2.38,2.36$ and 2.0$)$ to $1.91 \mathrm{~mm}(1.79,2.22$ and 1.71). Thereby the stiffness of the patellar tendon on average increased from $1795 \mathrm{~N} / \mathrm{mm}$ (1967, 727 and 2692) to $2519 \mathrm{~N} / \mathrm{mm}$ (3342, 883 and 3333) (Tab. 3, Fig. 1).

\section{Strength measures}

Mean isometric force before the intervention was 358 $\mathrm{N}(395,176$ and 504, respectively) and after the intervention $397 \mathrm{~N}$ (399, 178 and 614). Before the intervention, leg extensor power was $117 \mathrm{~W}$ (87, 68 and 197) and after, it was $123 \mathrm{~W}(115,74$ and 179). Three 5 RM tests were carried out for the lower body

Table 3. Biomechanical properties.

\begin{tabular}{llllllllll}
\hline & \multicolumn{2}{l}{ Participant 1 } & \multicolumn{2}{l}{ Participant 2 } & \multicolumn{2}{l}{ Participant 3 } & \multicolumn{2}{c}{ Total } \\
\hline & Pre & Post & Pre & Post & Pre & Post & Pre & Post & $\%$ \\
\hline Knee moment (N) & 96.2 & 104.2 & 32.7 & 41.4 & 122 & 167 & 83.7 & 104.2 & 23.9 \\
\hline Max def* (mm) & 2.38 & 1.79 & 2.36 & 2.22 & 2.00 & 1.71 & 2.25 & 1.91 & -14.9 \\
\hline Stiffness (N/mm) & 1967 & 3342 & 727 & 883 & 2692 & 3333 & 1795 & 2519 & 38.4 \\
\hline
\end{tabular}

$\%$ is the difference between pre and post expressed in percentage.

*Deformation: Max deformation is calculated from a common force value. 
Functional adaptation of tendon and skeletal muscle to resistance training in three patients with genetically verified classic Ehlers Danlos Syndrome

A) Force-deformation: Participant 1

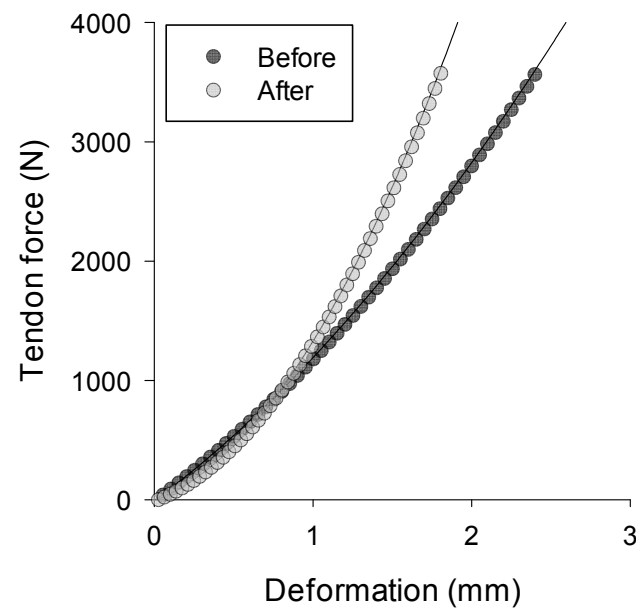

C) Force-deformation: Participant 3

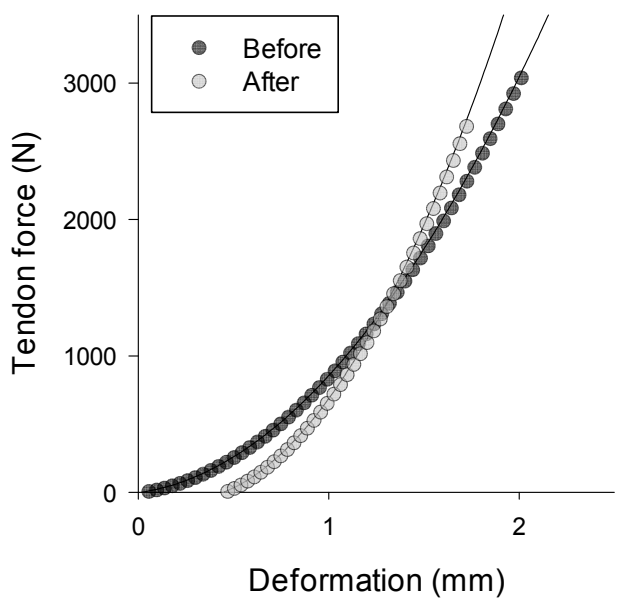

B) Force-deformation: Participant 2

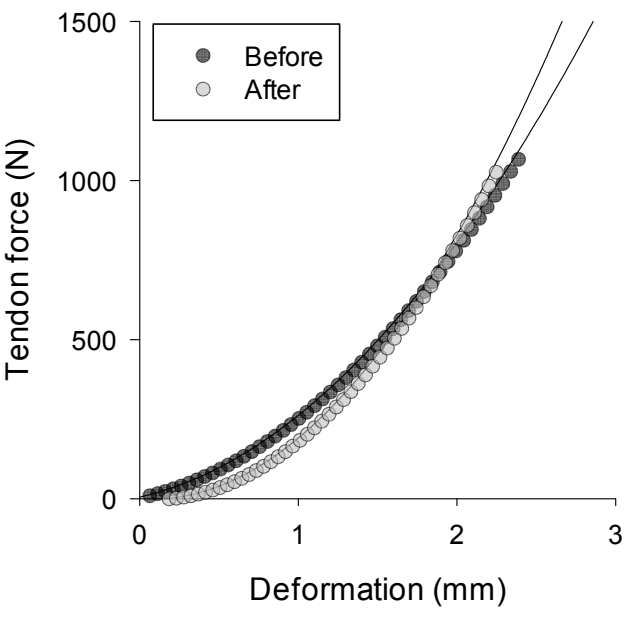

D) Stiffness

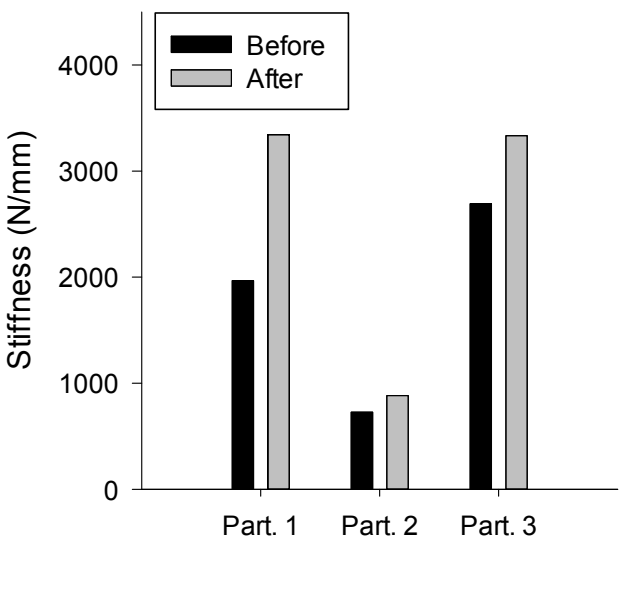

Figure 1. Force is measured by the dynamometer in the setup and deformation of the patellar tendon is measured in the ultrasound movie. The average values of two tests are used for the second order fitted regression line. Part., Participant

exercises and an average increase of $31 \%(37,28$ and 28) were observed. Increases were also detected for the upper body exercises. Training loads for these exercises were increased $34 \%(39,24$ and 39) from the $12^{\text {th }}$ to the $48^{\text {th }}$ training session (Tab. 4, Figs.2,3).

\section{Functional parameters}

In the chair rise test the participants completed 15 $(15,9$ and 21) rises before and 18 (16, 10 and 29) after the intervention. In the balance test, the sway area was $0.144 \mathrm{~m}^{2}(0.182,0.070$ and 0.178$)$ before the training and $0.108 \mathrm{~m}^{2}(0.093,0.049$ and 0.183$)$ after the training. In the CIS20 questionnaire of fatigue the participants scored 68 (78, 79 and 47$)$ in total and $33(41,40$ and 19) on the subscale of subjective fatigue before the intervention. After the intervention the participants scored $56(60,62$ and 46$)$ in total and $25(30,25$ and 19) on the subscale of subjective fatigue (Tab. 5, Fig. 4).

\section{Body composition}

The DEXA scans showed that the participants lost a total $2.1 \mathrm{~kg}$ of body weight over the training period $(+2.28,-1.99$ and -6.49$)$, of which $1.0 \mathrm{~kg}$ was lean mass $(+0.55,-2.62$ and -0.95$)$ and the fat percentage did not change (Tab. 5).

\section{Qualitative evaluation}

After the intervention the 3 participants who completed the training filled out an evaluation questionnaire in Danish (translated to English here). The questions were: 
Table 4. Strength measures.

\begin{tabular}{llllllllll}
\hline & \multicolumn{2}{l}{ Participant 1 } & \multicolumn{2}{l}{ Participant 2 } & \multicolumn{2}{l}{ Participant 3 } & \multicolumn{2}{c}{ Total } \\
\hline & Pre/12 & Post & Pre/12 & Post & Pre/12 & Post & Pre/12 & Post & $\%$ \\
\hline $\begin{array}{l}\text { Isometric leg ext. } \\
\text { force (N) }\end{array}$ & 395 & 399 & 176 & 178 & 504 & 614 & 358 & 397 & 7.97 \\
\hline Leg ext. power (W) & 87.0 & 115 & 68.0 & 74.0 & 197 & 179 & 117 & 123 & 10.6 \\
\hline 5 RM legext. (Kg.) & 40.0 & 47.0 & 30.0 & 42.0 & 75.0 & 95.0 & 48 & 61 & 28.1 \\
\hline 5 RM legcurl (Kg.) & 17.5 & 27.5 & 17.5 & -1 & 35.0 & 45.0 & 23.3 & $30^{1}$ & $28.6^{1}$ \\
\hline 5 RM legpress (Kg.) 110 & 150 & 110 & 160 & 220 & 280 & 147 & 197 & 36.4 \\
\hline Chest Press (Kg.) & $10.0^{*}$ & $15.0^{*}$ & $10.0^{*}$ & $10.0^{*}$ & $40.0^{*}$ & $55.0^{*}$ & $20.0^{*}$ & $26.7^{*}$ & 29.2 \\
\hline Seated Rows (Kg.) & $25.0^{*}$ & $35.0^{*}$ & $25.0^{*}$ & $27.5^{*}$ & $50.0^{*}$ & $62.5^{*}$ & $33.3^{*}$ & $41.7^{*}$ & 25.0 \\
\hline Abd. Crunch (Kg.) & $27.5^{*}$ & $40.0^{*}$ & $20.0^{*}$ & $32.5^{*}$ & $45.0^{*}$ & $80.0^{*}$ & $30.8^{*}$ & $50.8^{*}$ & 61.9 \\
\hline Lower b. ext. (Kg.) & $25.0^{*}$ & $30.0^{*}$ & $20.0^{*}$ & $25.0^{*}$ & $35.0^{*}$ & $60.0^{*}$ & $26.7^{*}$ & $38.3^{*}$ & 38.8 \\
\hline
\end{tabular}

The 5 RM parameters are results from the $5 \mathrm{RM}$ test conducted before and at the end of the study.

* The parameters of upper body strength are the training load at which participants performed the exercises in session $\mathrm{nr}$. 12 and in the last session before the tapering period.

$\%$ is the average difference between pre/12 anal and post values for each partecipant expressed in percentage.

1: Participant 2 wasn't able complete the post test. A post-value similar to the pre-value for participants was used for calculation of totals. Ext, extension; Abd., Abdominal; b, back.

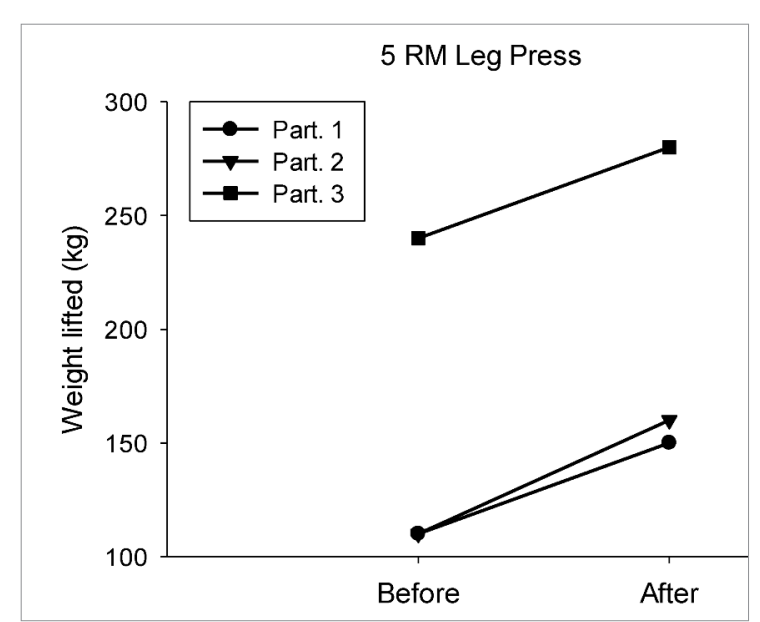

Figure 2. Strength test: 35 RM tests were performed before and after the intervention. The graph shows individual data from the leg press test. Before and after results for each participant are connected. Part., Participant.

A) Did you have more or less joint pain during the training compared to before starting the training? (More or Less). The participants answered the following 1: No change 2: No change (Comment: a lot more muscle pain during the training. The pain disappeared when the training stopped) 3: Less.

B) Did you have more or less joint pain after the training finished compared to before you started the training? (more or less) 1: No change (comment: Less back pain) 2: No change 3: Less.

C) Have you been more or less tired during the training period compared to before you started the

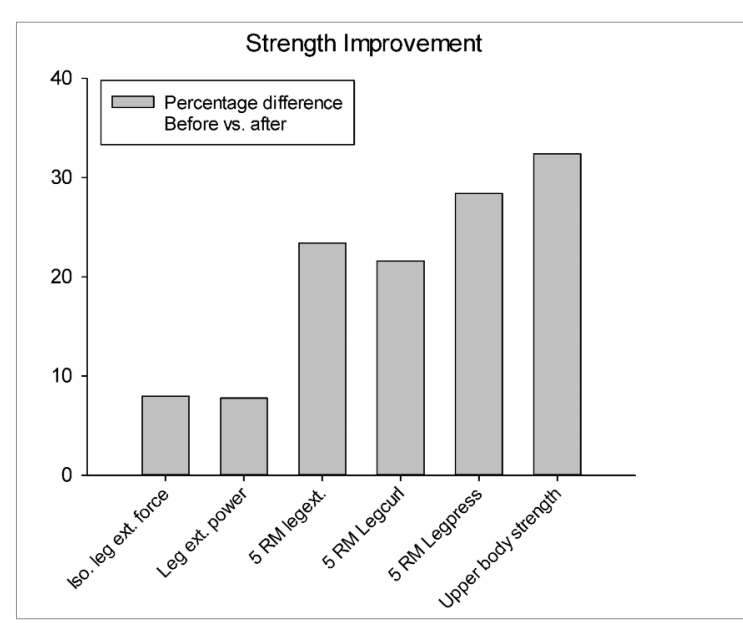

Figure 3. Strength parameters: Parameters from the strength tests are shown. The percentage differences are calculated as averages of the individual changes for the three participants. Iso. leg ext. force, Isometric leg extension force; ext., extension; 5 RM, 5 Repetition Maximum

training? (more or less). All 3 participants answered more.

D) Can you feel a physical change after you have completed the training intervention? (yes or no). All 3 participants answered yes. If yes, which? 1 : Less back pain, abdominals feels harder when contracted. 2: A little bit longer walking distance without crutches, better mobility and less pain in the right shoulder. 3: More strength, more energy, less sore, better shape

E) What do you think of training in relation to EDS? 1: Training is good! But it needs to be modified to 
Functional adaptation of tendon and skeletal muscle to resistance training in three patients with genetically verified classic Ehlers Danlos Syndrome

Table 5. Functional parameters and body composition.

\begin{tabular}{|c|c|c|c|c|c|c|c|c|c|}
\hline & \multicolumn{2}{|c|}{ Participant 1} & \multicolumn{2}{|c|}{ Participant 2} & \multicolumn{2}{|c|}{ Participant 3} & \multicolumn{3}{|c|}{ Total } \\
\hline & Pre & Post & Pre & Post & Pre & Post & Pre & Post & $\%$ \\
\hline \multicolumn{10}{|c|}{ Functional strength } \\
\hline 30 s. chair rise & 15.0 & 16.0 & 9.00 & 10.0 & 21.0 & 29.0 & 15.0 & 18.3 & 18.6 \\
\hline \multicolumn{10}{|l|}{ Balance } \\
\hline Sway area $\left(\mathrm{m}^{2}\right)$ & 0.182 & 0.093 & 0.070 & 0.049 & 0.178 & 0.183 & 0.144 & 0.108 & -25.5 \\
\hline \multicolumn{10}{|l|}{ Fatigue } \\
\hline CIS20 total & 78.0 & 60.0 & 79.0 & 62.0 & 47.0 & 46.0 & 68.0 & 56.0 & -15.6 \\
\hline Subj. Fatigue* & 41.0 & 30.0 & 40.0 & 25.0 & 19.0 & 19.0 & 33.3 & 24.7 & -21.4 \\
\hline \multicolumn{10}{|l|}{ Bodycomposition } \\
\hline Total tissue (kg.) & 55.9 & 58.2 & 73.5 & 71.5 & 80.7 & 74.2 & 70.0 & 68.0 & -2.23 \\
\hline L. b. mass (kg.) & 38.2 & 38.8 & 36.1 & 33.5 & 51.6 & 50.6 & 42.0 & 41.0 & -2.57 \\
\hline Tissue fat $\%$ & 31.7 & 33.4 & 50.8 & 53.1 & 36.1 & 31.7 & 39.5 & 39.4 & -0.77 \\
\hline
\end{tabular}

$\%$ is the average difference between pre and post expressed in percentage

* The subjective fatigue is a subscale of the CIS20 Checklist of Individual Strength containing 8 out of 20 items. Subj, Subjective; L. b. mass, Lean body mass

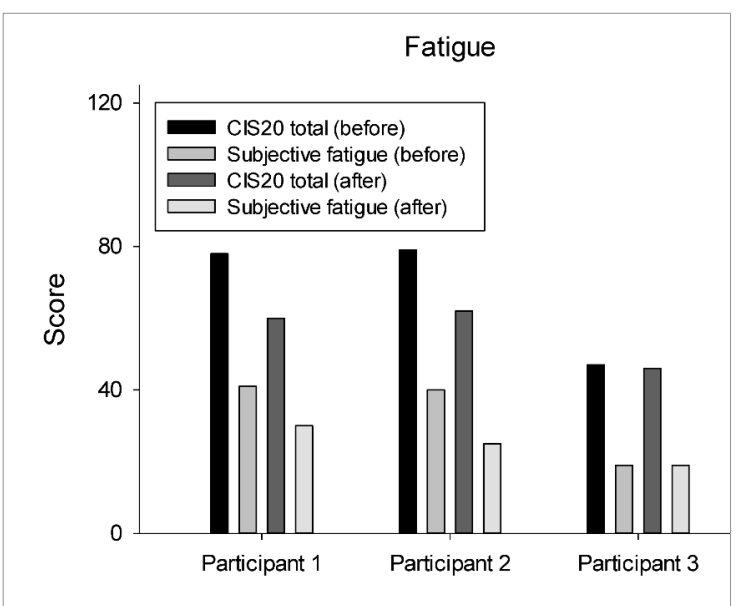

Figure 4. Fatigue: CIS20 Total and subscore values before and after the intervention are shown for each subject. The total score is based on all 20 items and the subscore is only based on those regarding subjective fatigue. Lower scores indicate lower general and subjective fatigue.

my needs. 2: Super - Should be offered to those who are physically capable. 3: Super nice, no doubt it has been beneficial.

F) Do you have ideas for changes or improvements? 1: Maximum twice a week. 2: Training two times a week and slower progression would have been better. 3: A couple of breaks of longer duration 3 times a week can be tough.

\section{Discussion}

This pilot study demonstrated that 3 patients with genetically verified classical Ehlers Danlos disease were able to complete a program with resistance muscle training without major side effects. All 3 train- ing participants reported minor occasional musculoskeletal or joint pain in some of the exercises. The training facility was located on the hospital where specialised clinicians would be able to take care of emerging symptoms, and this unfortunately limited some of the initial included subjects in their participation due to time consuming logistics. Although the low number of participants does not allow for a statistical evaluation of the outcome, it is interesting that training increased the tendon stiffness in the three individuals, in accordance with findings in healthy persons who undergo strength training 2,17 . This is interesting since it recently was demonstrated that patients with EDS have a lower stiffness of their tendons than both healthy and hypermobile counterparts, and suggests that despite the genetic error, the connective tissue in these patients is capable of adapting to physical training. Clearly, this needs confirmation in a full scale study. In addition to these observations, there was only a small increase in both the muscle isometric and power measures, whereas larger increases were observed with regards to the training load for both upper and lower body exercises.

The balance of women with the hypermobility type EDS has been studied and found to be impaired ${ }^{26}$, and it is therefore interesting that the sway area was reduced after strength training in the present study. It has previously been shown that fatigue is a major issue for patients suffering from EDS 27 , and that severe fatigue occur frequently in EDS and is correlated with pain ${ }^{28}$. A score of 76 for the full CIS20 questionnaire ${ }^{29}$ and 35 or more for the subscale of subjective fatigue ${ }^{28}$ has been set as the cut-off point for severe fatigue. Before the intervention participant 1 and 2 scored (78 and 41) and (79 and 40), respectively. This categorizes both participants as severely fatigued on both the total and the subscale of CIS20. After the intervention the 2 participants scored $(60$ and 30 ) and (62 and 25), respectively. In this study, none of the participants were any longer categorized 
as severely fatigued after the end of the intervention. On the other hand, no changes in fatigue scores were seen in participant 3 , which could be due to the lower starting point (47 and 19). As mentioned in the introduction, fatigue is correlated with muscle weakness in EDS patients ${ }^{14}$. The explanation for the lowered fatigue score in the 2 subjects could be increased muscle strength, but studies will have to prove this hypothesis. The authors are aware of the fact, that symptoms and capabilities of patients with EDS are very different. This is why resistance training is possibly not an option for all patients suffering from EDS. Furthermore, to get the best outcome for each individual it could be optimal to tailor an individual programme for every person to assure that problematic joints are targeted in the most optimal way, and in this perspective the present observations are only case studies.

In a recent review ${ }^{30}$, guidelines to manage symptoms of pain and fatigue in EDS are given. The first two lifestyle recommendations concerning pain and fatigue in BJHS/htEDS given by Castori et al. are "Promote regular, aerobic fitness" and "promote fitness support with strengthening, gentle stretching and proprioception exercises". On the other hand it is in the same lifestyle recommendations recommended to "avoid excessive weight lifting/carrying". Clearly the picture on whether to recommend or avoid heavy resistance training is not evident.

Therefore it is crucial to perform larger controlled studies to investigate if EDS patients have a blunted response to resistance training compared to healthy subjects and if this is not the case to investigate if increased strength can benefit patients on parameters such as joint stability, pain, fatigue and quality of daily life.

\section{Conclusion}

The authors can conclude that heavy resistance training was both feasible and safe in three patients with genetically verified connective tissue defects (classic Ehlers Danlos Syndrome). The participants demonstrated improved musculo-tendinous function, and somewhat lower fatigue as a result of the training ${ }^{31}$.

\section{Declaration of interest}

The authors report no conflicts of interest. This study was supported through public and private research foundation grants given to the Institute of Sports Medicine Copenhagen.

\section{Acknowledgement}

The authors thank Christina Hansen for performing the biomechanical tests and analysing the data. The authors also thank Jonas Frimer who has helped with analyzes.

\section{References}

1. Kraemer WJ, Ratamess NA, French DN. Resistance training for health and performance. Curr Sports Med Rep. 2002;1(3):165-171.

2. Couppe $\mathrm{C}$, Kongsgaard $\mathrm{M}$, Aagaard $\mathrm{P}$, et al. Habitual loading results in tendon hypertrophy and increased stiffness of the human patellar tendon. J Appl Physiol (1985). 2008;105(3):805810.

3. Boesen AP, Dideriksen K, Couppe C, et al. Tendon and skeletal muscle matrix gene expression and functional responses to immobilisation and rehabilitation in young males: effect of growth hormone administration. J Physiol. 2013;591(Pt 23):6039-6052.

4. Boesen AP, Dideriksen K, Couppe C, et al. Effect of growth hormone on aging connective tissue in muscle and tendon: gene expression, morphology, and function following immobilization and rehabilitation. J Appl Physiol (1985). 2014;116(2):192-203.

5. Couppe C, Suetta C, Kongsgaard M, et al. The effects of immobilization on the mechanical properties of the patellar tendon in younger and older men. Clin Biomech (Bristol, Avon). 2012;27(9):949-954.

6. Beighton P, De Paepe A, Steinmann B, Tsipouras P, Wenstrup RJ. Ehlers-Danlos syndromes: revised nosology, Villefranche, 1997. Ehlers-Danlos National Foundation (USA) and Ehlers-Danlos Support Group (UK). Am J Med Genet. 1998;77(1):31-37.

7. Steinmann B, Royce PM, Superti-Furga A. The Ehlers-Danlos Syndrome. In: Steinmann PMRaB, editor. Connective Tissue and Its Heritable Disorders: Molecular, Genetic, and Medical Aspects. Second Edition. Hoboken, NJ, USA. John Wiley \& Sons, Inc.; 2003.

8. Castori M. Ehlers-danlos syndrome, hypermobility type: an underdiagnosed hereditary connective tissue disorder with mucocutaneous, articular, and systemic manifestations. ISRN Dermatol. 2012;2012:751768.

9. Symoens S, Syx D, Malfait F, et al. Comprehensive molecular analysis demonstrates type $\mathrm{V}$ collagen mutations in over $90 \%$ of patients with classic EDS and allows to refine diagnostic criteria. Hum Mutat. 2012;33(10):1485-1493.

10. Nielsen RH, Couppe C, Jensen JK, et al. Low tendon stiffness and abnormal ultrastructure distinguish classic Ehlers-Danlos syndrome from benign joint hypermobility syndrome in patients. FASEB J. 2014.

11. Malfait F, Wenstrup RJ, De Paepe A. Clinical and genetic aspects of Ehlers-Danlos syndrome, classic type. Genet Med. 2010;12(10):597-605.

12. Malfait F, Wenstrup R, De Paepe A. Ehlers-Danlos Syndrome, Classic Type. In: Pagon RA, Adam MP, Ardinger HH, Bird TD, Dolan CR, Fong CT, et al., editors. GeneReviews(R). Seattle (WA) 1993.

13. Voermans NC, Knoop H, Bleijenberg G, van Engelen BG. Pain in ehlers-danlos syndrome is common, severe, and associated with functional impairment. J Pain Symptom Manage. 2010;40(3):370-378.

14. Voermans NC, Knoop H, Bleijenberg G, van Engelen BG Fatigue is associated with muscle weakness in Ehlers-Danlos syndrome: an explorative study. Physiotherapy. 2011;97(2):170-174.

15. Kraemer WJ, Ratamess NA. Fundamentals of resistance training: progression and exercise prescription. Med Sc Sports Exerc. 2004;36(4):674-688.

16. Brzycki M. predicting a one-rep max from reps to fatigue. JOPERD. 1993;64(64):88-90.

17. Couppe $\mathrm{C}$, Kongsgaard $\mathrm{M}$, Aagaard $\mathrm{P}$, et al. Differences in tendon properties in elite badminton players with or without patellar tendinopathy. Scand J Med Sci Sports. 2013;23(2):e89-95. 
Functional adaptation of tendon and skeletal muscle to resistance training in three patients with genetically verified classic Ehlers Danlos Syndrome

18. Hansen $\mathrm{P}$, Bojsen-Moller $\mathrm{J}$, Aagaard $\mathrm{P}$, Kjaer M, Magnusson SP. Mechanical properties of the human patellar tendon, in vivo. Clin Biomech (Bristol, Avon). 2006;21(1):54-58.

19. Maffiuletti NA, Bizzini M, Widler K, Munzinger U. Asymmetry in quadriceps rate of force development as a functional outcome measure in TKA. Clin Orthop Relat Res. 2010;468(1):191-198.

20. Rantanen T, Era P, Heikkinen E. Physical activity and the changes in maximal isometric strength in men and women from the age of 75 to 80 years. J Am Geriatr Soc. 1997;45(12):1439-1445.

21. Narici MV, Landoni L, Minetti AE. Assessment of human knee extensor muscles stress from in vivo physiological cross-sectional area and strength measurements. Eur J Appl Physiol Occup Physiol. 1992;65(5):438-444

22. Bassey EJ, Fiatarone MA, O'Neill EF, Kelly M, Evans WJ, Lipsitz LA. Leg extensor power and functional performance in very old men and women. Clin Sci (Lond). 1992;82(3):321327.

23. Jones CJ, Rikli RE, Beam WC. A 30-s chair-stand test as a measure of lower body strength in community-residing older adults. Res Q Exerc Sport. 1999;70(2):113-119.

24. Rikli RE, Jones CJ. Development and Validation of a functional fitness test for community-residing older adults. J Aging Phys. 1999(Act. 7):127-159.

25. Vercoulen JH, Swanink CM, Fennis JF, Galama JM, van der
Meer JW, Bleijenberg G. Dimensional assessment of chronic fatigue syndrome. J Psychosom Res. 1994;38(5):383-392.

26. Rombaut L, Malfait F, De Wandele I, et al. Balance, gait, falls, and fear of falling in women with the hypermobility type of Ehlers-Danlos syndrome. Arthritis Care Res (Hoboken). 2011;63(10):1432-1439.

27. Gawthrop F, Mould R, Sperritt A, Neale F. Ehlers-Danlos syndrome. BMJ. 2007;335(7617):448-450.

28. Voermans NC, Knoop H, van de Kamp N, Hamel BC, Bleijenberg $G$, van Engelen BG. Fatigue is a frequent and clinically relevant problem in Ehlers-Danlos Syndrome. Semin Arthritis Rheum. 2010;40(3):267-274.

29. Bultmann U, de Vries M, Beurskens AJ, Bleijenberg G, Vercoulen $\mathrm{JH}$, Kant I. Measurement of prolonged fatigue in the working population: determination of a cutoff point for the checklist individual strength. J Occup Health Psychol. 2000;5(4):411-416.

30. Castori M, Morlino S, Celletti C, et al. Management of pain and fatigue in the joint hypermobility syndrome (a.k.a. Ehlers-Danlos syndrome, hypermobility type): principles and proposal for a multidisciplinary approach. Am J Med Genet A. 2012;158A(8):2055-2070.

31. Padulo J, Oliva F, Frizziero A, Maffulli N. Muscles, Ligaments and Tendons Journal. Basic principles and recommendations in clinical and field science research. MLTJ. 2013;4:250-252. 